\title{
Le droit viti-vinicole au Japon en tant que pont entre les producteurs et les consommateurs
}

\author{
Kensuké Ebihara $^{1}$, Makiko Omura ${ }^{2}$, et Yuka Sakuraï ${ }^{3}$ \\ ${ }^{1}$ Faculté de droit, Université Meijigakuin de Tokyo, 108-8636, Tokyo, Japon \\ ${ }^{2}$ Faculté d'économie, Université Meijigakuin de Tokyo, 108-8636, Tokyo, Japon \\ ${ }^{3}$ Faculté de gestion, Université Rikkyo (Saint Paul), 171-8501, Tokyo, Japon
}

\begin{abstract}
Résumé. Le droit viti-vinicole au Japon est caractérisé par son insuffisance, car il n'existe pas de définition législative du vin, ni règlementation juridique sur l'étiquetage. Cependant, en 2014, le parti libéral démocrate, a annoncé son intention de légiférer une loi sur le vin et l'Agence de la taxe nationale examine, en ce moment, la possibilité d'améliorer les normes existantes. Avec l'enregistrement de l'indication géographique viticole et la mise en vigueur de la loi relative à la protection du nom géographique des produits agroalimentaires, on observe l'émergence d'un pont entre les producteurs et les consommateurs.
\end{abstract}

\section{Développement du marché viticole au Japon}

\subsection{Pays producteur et consommateur}

Le Japon est un pays producteur et consommateur de vin. En 2013, la consommation totale au Japon a dépassé 3 millions hl, alors que la consommation per capita reste relativement faible, soit 3,1 litres par an, par rapport aux principaux pays membres de l'OIV.

En ce qui concerne la production de vin, il existe deux catégories différentes de vin produit au Japon : premièrement, « le vin japonais » issu des raisins récoltés au Japon et deuxièmement, « le vin fermenté au Japon » issu de moût ou de jus de raisin importé.

Selon une enquête effectuée par l'Agence de la taxe nationale, plus de 75 pour cent du vin « fabriqué au Japon » utilise le moût de raisin importé, essentiellement en provenance d'Amérique de Sud. Cependant, il nous semble que cette catégorie de produits est nécessaire afin de commercialiser les vins moins chers pour les consommateurs modestes.

\subsection{Augmentation importante de du nombre de exploitations au Japon}

A partir du 19ème siècle, le Japon a commencé la production de vin dans certaines régions viticoles. En 2015, il existe plus de 210 exploitations viticoles. Les régions les plus représentatives sont Yamanashi (79 exploitations), Nagano (27 exploitations), Hokkaido (24 exploitations) et Yamagata (12 exploitations).

Il est assez étonnant que, depuis une dizaine années, le nombre de nouveaux entrants augmente de façon considérable. On observe les augmentations les plus importantes dans les préfectures de Nagano et Hokkaido.
L'avantage de Hokkaido consiste dans la disponibilité de terrain utilisable, son prix raisonnable et une pluviométrie relativement faible.

Quant à Nagano, l'autorité locale, elle-même, s'engage dans le développement des zones viticoles. La préfecture de Nagano a présenté, en 2013, «Shinshu Wine Valley Project », un plan préfectoral pour le développement des zones viticoles dans la préfecture de Nagano. Ce plan stratégique a pour but de réaliser l'augmentation de production de vin issue de raisins de cuve, notamment l'espèce Vitis vinifera, l'amélioration de la qualité du vin, la valorisation du vin de la région ainsi que la promotion du tourisme vitivinicole et œnologique.

\subsection{Boom du vin japonais}

Malgré la quantité importante de vin importé sur le marché japonais, les consommateurs ont tendance à préférer le vin japonais.

Le boom du vin japonais est accéléré par l'amélioration considérable de sa qualité. Depuis 2003, la préfecture de Yamanashi organise, chaque année, la «Japan Wine Competition », un concours national du vin japonais. Afin de figurer au palmarès, les producteurs, y compris les viticulteurs familiaux, multiplient leurs efforts.

La loi Evin n'existe pas au Japon. Les chaînes TV diffusent des programmes qui peuvent promouvoir la vente du vin japonais en fournissant des informations sur les producteurs et leurs produits. Certains producteurs ont lancé une campagne publicitaire sur l'accord avec les cuisines régionales. Grâce à l'accessibilité auprès des producteurs, les consommateurs peuvent facilement recueillir des informations et approfondir leurs connaissances. 
Tableau 1. Evolution de la consommation de vin au Japon.

\begin{tabular}{|l|l|l|l|}
\hline & vin non importé & vin importé & total (hl) \\
\hline 1987 & 510810 & 293620 & 804430 \\
\hline 1992 & 589370 & 510210 & 1099580 \\
\hline 1997 & 1085660 & 1162080 & 2247740 \\
\hline 2002 & 1017990 & 1572320 & 2590310 \\
\hline 2007 & 780390 & 1514880 & 2952700 \\
\hline 2012 & 920650 & 2287200 & 3207850 \\
\hline
\end{tabular}

Source : Agence de la taxe nationale.

Tableau 2. Evolution du nombre de nouvelles exploitations viticoles.

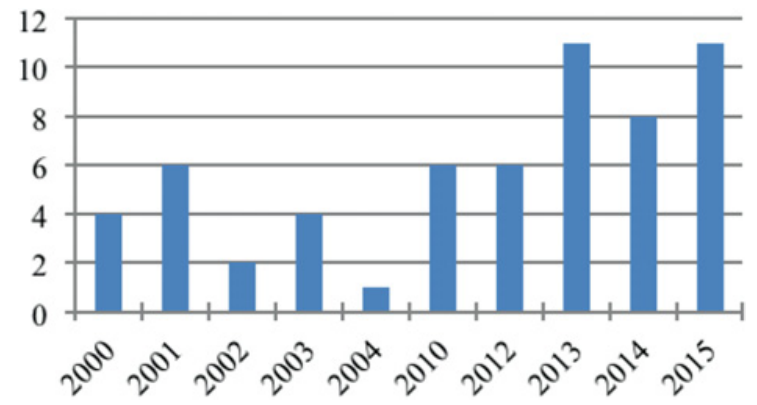

Tableau 3. Production de raisin de cuve en 2010 (en tonnes).

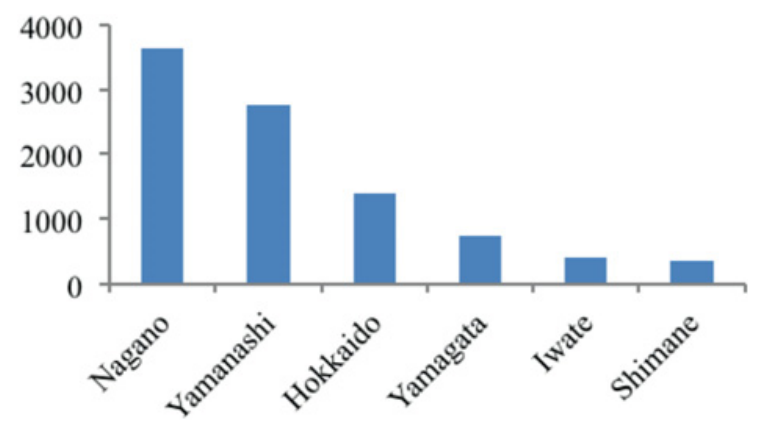

Source : ministère de l'Agriculture.

\section{Difficulté juridique}

\subsection{Absence de législation nationale}

Le Japon est un pays producteur et même exportateur, cependant il n'existe pas de définition législative du vin, ni règlementation juridique sur l'étiquetage.

En l'absence de législation nationale, la distinction juridique entre ces deux catégories, « le vin japonais » et 《le vin fermenté au Japon », n'est pas clairement établie. Il est évident que cette situation empêche d'établir un pont entre les producteurs et les consommateurs.

Bien que l' « Association japonaise de producteurs de vin $»$, un groupement national des producteurs, ait rédigé un règlement intérieur, modifié en 2006, sur l'étiquetage du vin, le nombre de producteurs adhérant à l'association est très limité, seulement 43 membres, alors que l'on compte plus de deux cent exploitations viticoles sur l'archipel.

\subsection{Débats sur la nécessité de législation nationale}

En 2014, le parti libéral démocrate, le parti au pouvoir, a annoncé son intention de légiférer une loi sur le vin. Selon des sénateurs du parti libéral démocrate, l'absence de législation nationale empêche la valorisation de vin japonais sur le marché international, surtout dans les pays membres de l'UE qui dispose une réglementation très stricte. En vue de légiférer la loi sur le vin, ils ont organisé un groupe d'études.

Par contre, les producteurs ont exprimé une inquiétude, car la réglementation stricte n'est pas convenable pour la viticulture au Japon. A la différence du marché européen, qui souffre de la surproduction, la difficulté au Japon consiste dans le manque quantitatif de raisin de cuve pour la production de vin. Les producteurs craignent la diminution de production et l'uniformisation des vins, car la diversité du vin japonais est essentielle pour attirer l'attention des consommateurs.

\subsection{Vers amélioration des normes existantes}

L'Agence de la taxe nationale, l'autorité compétente dans le secteur, commence, quant à elle, à examiner la possibilité d'améliorer les normes existantes. Par exemple, l'Agence a admis qu'il est inévitable de définir, de manière claire, « le vin japonais », car il n'existe aucune définition juridique aujourd'hui. Les conditions de l'enregistrement d'une indication géographique sont également en question.

De même, l' « Association japonaise de producteurs de vin », prépare une modification du règlement intérieur concernant l'étiquetage du vin suivant la norme adoptée par l'UE.

\section{Evolution récente de protection de l'indication géographique}

\subsection{Enregistrement de l'indication géographique viticole}

Il est probable que le système national de l'indication géographique peut servir de pont entre les producteurs et les consommateurs. Après l'enregistrement, pour la première fois en juillet 2013, par l'Agence de la taxe nationale, de l'indication géographique viticole «Yamanashi », les producteurs doivent respecter le cahier des charges pour l'utilisation du nom géographique « Yamanashi », un nom désormais juridiquement protégé. La mise en valeur du système de l'indication géographique est primordiale afin de fournir des informations nécessaires aux consommateurs japonais et aussi aux consommateurs des pays membres de l'OIV.

A la suite de l'enregistrement de « Yamanashi », l'association de producteurs de vins de Hokkaido a décidé, en février 2015, de se lancer dans l'enregistrement d'une nouvelle indication géographique «Hokkaido » afin de protéger son nom géographique et la notoriété du vin de Hokkaido. Le cahier des charges, qui peut concerner de nombreux producteurs, n'est pas encore publié. Il est possible qu'il interdise l'utilisation de l'indication géographique en question à des vins élaborés à l'extérieur 
Tableau 4. Cahier des charges de l'IG Yamanashi.

\begin{tabular}{|l|l|}
\hline $\begin{array}{l}\text { Dénomination } \\
\text { géographiques }\end{array}$ & Yamanashi \\
\hline Récolte des raisins & $\begin{array}{l}\text { toutes les communes } \\
\text { dans la préfecture de } \\
\text { Yamanashi }\end{array}$ \\
\hline $\begin{array}{l}\text { Vinification, élaboration et } \\
\text { conditionnement }\end{array}$ & $\begin{array}{l}\text { toutes les communes } \\
\text { dans la préfecture de } \\
\text { Yamanashi }\end{array}$ \\
\hline Encépagement & $\begin{array}{l}\text { espèce Vitis vinifera, } \\
\text { Koshu, Muscat Bailey A, } \\
\text { Black Queen, Bailey Ali- } \\
\text { cante A } \\
\text { Kai Noir, Kai Blanc, } \\
\text { Sun Semillon, Delaware }\end{array}$ \\
\hline $\begin{array}{l}\text { Richesse minimale en sucre } \\
\text { des raisins ( }{ }^{\circ} \text { B) }\end{array}$ & $\begin{array}{l}\text { espèce Vitis vinifera : 18,0 } \\
\text { Koshu : 14,0 } \\
\text { autres variétés autorisées : } \\
16,0\end{array}$ \\
\hline $\begin{array}{l}\text { Titre alcoométrique mini- } \\
\text { mum }\end{array}$ & $\begin{array}{l}\text { vins secs : } 8,5 \% \\
\text { vins doux : 4,5\% }\end{array}$ \\
\hline Examen organoleptique & obligatoire \\
\hline Responsable de contrôle & $\begin{array}{l}\text { l'Agence de la taxe na- } \\
\text { tionale }\end{array}$ \\
\hline
\end{tabular}

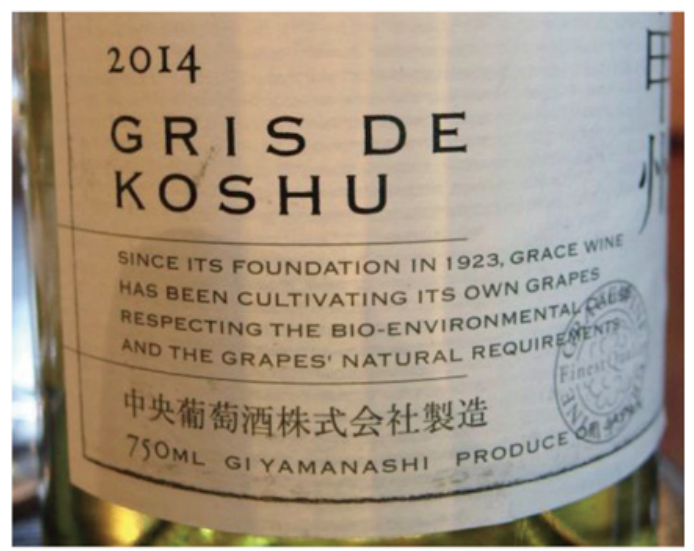

Figure 1. Mention de l'indication géographique sur l'étiquette.

de la préfecture de Hokkaido. En réalité, certains producteurs utilisent les raisins récoltés à Hokkaido, mais élaborent leurs vins dans d'autres préfectures telles que Yamanashi, Okayama et Tochigi.

Le groupement de viticulteurs de Nagano, lui aussi, a exprimé son intention de protéger son nom géographique en utilisant le système national de l'indication géographique.

\subsection{Protection de l'indication géographique des produits agroalimentaires}

En juin 2014, le parlement japonais a voté, presque unanimement, la loi relative à la protection du nom géographique des produits agroalimentaires, forestiers et maritimes, c'est-à-dire la loi sur l'indication géographique. Il est évident que la grande majorité des dispositions de la loi est inspirée par le règlement européen sur l'indication géographique.

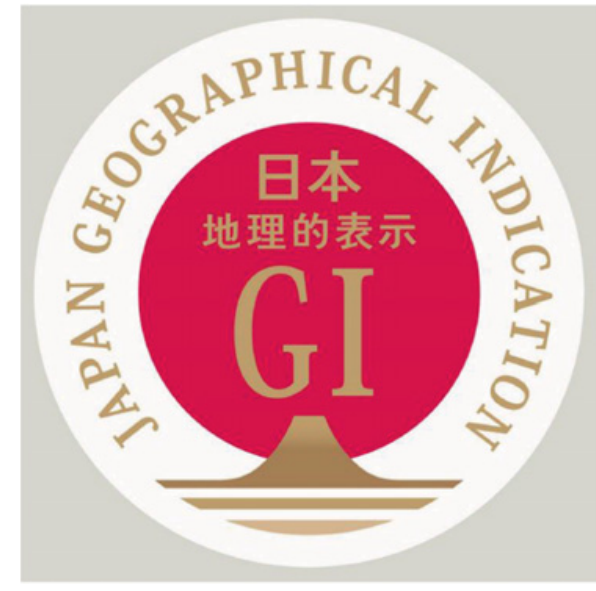

Figure 2. Signe national de l'indication géographique.

Comme cette loi est du ressort du ministère de l'Agriculture, elle n'est pas applicable aux boissons alcoolisées, y compris le vin et le saké. Au Japon, c'est l'Agence de la taxe nationale qui est compétente en la matière. Toutefois, la mise en valeur de la loi peut permettre de faire pénétrer efficacement la notion de l'indication géographique dans l'esprit des consommateurs japonais.

\section{Conclusion}

Même si le droit vitivinicole au Japon comporte des insuffisances, on peut remarquer l'émergence du pont entre les producteurs et les consommateurs dans le contexte de l'introduction du système de l'indication géographique et l'initiative politique et administrative en vue d'améliorer les règles sur l'étiquetage du vin japonais. Cependant, pour que le pont soit solide, il faut que le Japon adhère à l'OIV. L'adhésion à l'OIV est donc inévitable pour renforcer les liens entre les producteurs et les consommateurs en fournissant des informations adéquates en la matière.

\section{Références}

K. Ebihara, « L'impact de la réforme de l'OCM vitivinicole : l'exemple japonais », Rivista di diritto alimentare, 2008-4 (2008)

K. Ebihara, M. Omura, S. Hirakawa, K. Hasegawa, « L'impact des indications géographiques d'origine sur la consommation de vin au Japon », Le Bulletin de l'OIV, 82, 944-945-946 (2009)

K. Ebihara, «Que font-ils ailleurs ? : Évolution des règles sur l'étiquetage des vins au Japon », Revue des Oenologues, 131 (2009)

H. Yamamoto, T. Takahashi, K. Ebihara, Le droit vitivinicole comparé, (Editions Nippon Hyoron Sha, 2009)

K. Ebihara, M. Omura, S. Hirakawa, K. Hasegawa, « Le indicazioni geografiche di origine ed il consumo del vino in Giappone », OICCE TIMES, 43 (2010)

$\mathrm{K}$. Ebihara, Introduction au droit viti-vinicole, (Editions Kohyusha, 2014) 